\title{
Measurement of Mobile Switching Centres Throughput in GSM Network Integrating Sliding Window Algorithm with a Single Server Finite Queuing Model
}

\author{
Dinaker Babu Bollini, ${ }^{1}$ Mannava Muniratnam Naidu, ${ }^{2}$ and Mallikharjuna Rao Nuka ${ }^{3}$ \\ ${ }^{1}$ Department of Computer Science \& Engineering, SVU College of Engineering, Tirupati, Andhra Pradesh 517502, India \\ ${ }^{2}$ Department of Computer Science and Engineering, RVR \& JC College of Engineering, Guntur, Andhra Pradesh 522019, India \\ ${ }^{3}$ Department of Computer Applications, Annamacharya Institute of Technology \& Sciences, Rajampet, Andhra Pradesh, India \\ Correspondence should be addressed to Dinaker Babu Bollini; dinaker@hotmail.com
}

Received 9 April 2016; Revised 25 August 2016; Accepted 8 September 2016

Academic Editor: Lixin Gao

Copyright ( 2016 Dinaker Babu Bollini et al. This is an open access article distributed under the Creative Commons Attribution License, which permits unrestricted use, distribution, and reproduction in any medium, provided the original work is properly cited.

\begin{abstract}
The sliding window algorithm proposed for determining an optimal sliding window does not consider the waiting times of call setup requests of a mobile station in queue at a Mobile Switching Centre (MSC) in the Global System for Mobile (GSM) Communication Network. This study proposes a model integrating the sliding window algorithm with a single server finite queuing model, referred to as integrated model for measurement of realistic throughput of a MSC considering the waiting times of call setup requests. It assumes that a MSC can process one call setup request at a time. It is useful in determining an optimal sliding window size that maximizes the realistic throughput of a MSC. Though the model assumes that a MSC can process one call setup request at a time, its scope can be extended for measuring the realistic throughput of a MSC that can process multiple call setup requests at a time.
\end{abstract}

\section{Introduction}

A mobile subscriber is uniquely identified by Mobile Station International Subscriber Directory Number (MSISDN) in the Global System for Mobile (GSM) Communication Network. The GSM network provides services to its registered mobile subscribers using the Gateway Mobile Switching Centre (GMSC) of a service provider. The GMSC area is partitioned into several areas with a switching centre at each area referred to as Mobile Switching Centre (MSC) connected to GMSC. The profiles of mobile subscribers are stored persistently in Home Location Register (HLR) at GMSC. Each MSC maintains a Visitor Location Register (VLR), which stores the profiles of mobile subscribers temporarily [1]. A MSC provides call setup services in response to a call setup request from a mobile subscriber provided its profile is available in VLR.

The mobile subscribers roam randomly in the GSM network area. It is observed that the policy of accessing HLR is fetching the profile of a mobile subscriber to VLR whenever it arrives at MSC service area and deleting the same whenever it leaves. This method increases the load on the network traffic, delays call setup time, and reduces the throughput of MSC [2]. Shah Newaz et al. [3] proposed a method of Fixed Block of Seven Days (FBSD) for determining the retention period of the profile of a mobile subscriber in VLR for improving the throughput of a MSC. Nuka and Naidu [4] proposed a sliding window of size seven days (SWSSD) algorithm which proved to be better than that of FBSD. This model partitions the sliding window into seven slots and maps onto seven days.

At the end of each day beyond initial seven days, the model determines the intersection of mobile subscribers' profiles over seven slots, slides the window by one slot right masking the front slot and opening new slot at the other end, and copies the intersection to newly opened slot.

A simulation model is developed for evaluating the performance of FBSD and SWSSD algorithms. The analysis of simulation output proved that the SWSSD algorithm is better than the existing FBSD algorithm as it decreases the average call setup time by $6.28 \%$ and increases the 
throughput by $7.08 \%$. Further, Nuka and Naidu [5] proposed a model for determining optimal sliding window size (OSWS) considering the average call setup time and throughput as criteria of optimization.

However, the SWSSD algorithm [4] has not considered the waiting time of call setup requests in queue at a MSC when it is busy. This study proposes a model integrating SWSSD algorithm with a single server finite queuing model (SSFQM) referred to as an integrated model (IM) for realistic measurement of throughput of a MSC considering the waiting time of call setup requests in queue.

The paper is divided into 8 sections inclusive of this section. In Section 2, a brief review of related work is presented. Section 3 defines the problem. An outline of SWSSD is presented in Section 4. The SSFQM is presented in Section 5. Section 6 presents the integration of SWSSD algorithm with SSFQM. The process of measuring the realistic throughput of a MSC through simulation is presented in Section 7. The conclusions of the study are enumerated in Section 8.

\section{Review of Related Work}

GSM network system is portioned into three subsystems, namely, Base Station Subsystem (BSS), Network Switching Subsystem (NSS), and Operational Subsystem (OSS) as shown in Figure 1. The BSS routes the call setup request of a mobile subscriber to NSS. A cell is an area of a GSM network in which the subscriber can connect to a Base Transceiver Station (BTS) through radio signals to transmit data. A set of cells in the jurisdiction of a BTS is known as a location area (LA). A set of BTSs are connected to a Base Station Controller (BSC). The NSS provides the call setup service in response to the request. The BSS and NSS utilize the services of OSS for providing their requisite services to mobile subscriber for materializing conversation among mobile subscribers. Obviously, it is typical that mobile subscribers roam randomly over location areas.

The profiles of all the mobile subscribers registered with a service provider are maintained using a centralized database at GMSC referred to as HLR. Whenever a MSC receives call setup request from a mobile subscriber through the BSS, it processes it for providing call setup services. The availability of profile of mobile subscriber at the MSC is necessary for processing the call setup request. To meet this prerequisite, at each MSC a database, referred to as VLR, is maintained for storing temporarily the profiles of mobile subscribers which are currently in the service area of a MSC. One extreme policy is replicating the HLR as VLR at each MSC. This policy increases throughput of a MSC while increasing the cost of storage. Other extreme policy is fetching the profile whenever the mobile subscriber enters the location area and deleting the same on its exit. This policy decreases the throughput of MSC while increasing network traffic overhead. Hence, it is essential to undertake the problem for determining the retention period of the profiles of mobile subscribers in VLR in spite of leaving the service area of a MSC with the objective of maximizing the throughput of MSC while minimizing the sum of the costs of storage and network traffic overhead.

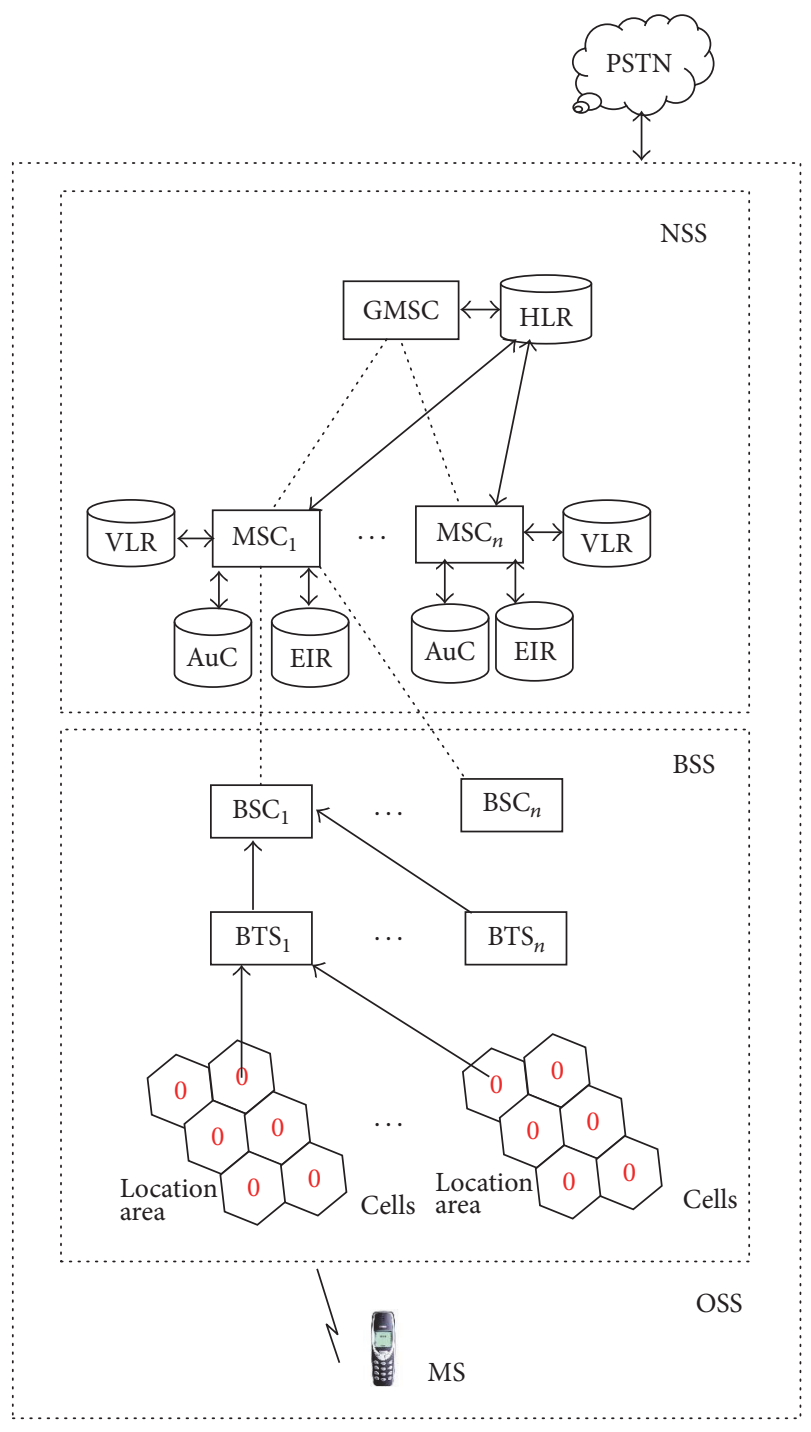

FIGURE 1: GSM network architecture.

The review of related work reported in [6-16] has not addressed this problem. This problem is addressed in [3] proposing a Fixed Block of Seven Days (FBSD) model for determining the retention period of the profile in VLR for improving the throughput of a MSC. Subsequently, Nuka and Naidu [4] proposed sliding window of size seven days algorithm that proved better than the above-said model [3]. Further, they extended the algorithm for determining optimal sliding window size [5] that maximizes throughput of MSC.

However, the SWSSD algorithm $[4,5]$ has not considered the waiting time of call setup requests in queue at a MSC when it is busy. Therefore, it is motivated to propose a model integrating SWSSD algorithm with a single server finite queuing model (SSFQM) for measurement of realistic throughput of a MSC considering call setup request waiting in queue. 


\section{Problem Statement}

A model proposed in [5] for determining an optimal sliding window size has not considered the waiting time of call setup requests in queue due to busy state of a MSC. It is essential to consider the waiting time of call setup requests for formulating a more realistic model. Hence, formulating a model for measuring the throughput of a MSC considering waiting time of call setup requests in queue due to busy state of a MSC is a prerequisite for determining an optimal sliding window size. Further, such a model can also be used for developing a cost model for determining an optimal number of identical channels for a MSC. Therefore, it is motivated to undertake the problem of measuring throughput of a MSC with a single channel integrating sliding window of size seven days algorithm with a single server finite queuing model.

\section{Sliding Window of Size Seven Days Algorithm}

The SWSSD algorithm has been proposed for determining the holding period of a record of a mobile subscriber profile in VLR, instead of deleting immediately when the mobile subscriber leaves the service area of a MSC, with an objective of maximizing throughput of the MSC and minimizing call setup time [4]. Here, the overall logic of the algorithm is briefly described.

On a given day, the MSISDNs made call setup requests which comprise one set of the sliding windows. Initially, the days are denoted by numbers one to seven and their corresponding sets are set to null. A set is updated applying union operation between the current set and a MSISDN from which the call setup request is received, provided the relevant record of the MSISDN is fetched from HLR due to nonavailability of the same in VLR. At end of the seventh day, the intersection of seven sets is determined. The set in the first position of sliding window is deleted by shifting other six sets left by one position. This is equivalent to sliding the window to right by one position. The intersection is copied in the seventh position of sliding window. Consequently, the days are incremented by one. Thereafter, at the end of each day, the tasks of determining the intersection, sliding the window, copying the intersection, and incrementing days are repeated over days. The definitions of notation are given below:

$$
\begin{aligned}
S_{i} & =\{s \mid s \text { is an MSISDN that makes at least one call setup request on } i \text { th day }\} \quad \text { for }(1 \leq i \leq 7), \\
\mathrm{fp} & =\text { day of first position of window, } \\
\mathrm{sp} & =\text { day of seventh position of window, } \\
I & =\bigcap_{i=\mathrm{fp}}^{i=\mathrm{pp}} S_{i} .
\end{aligned}
$$

The pseudocodes for call setup process procedure and sliding window algorithm are presented in Algorithms 1 and 2, respectively.

The inputs to CALLSETUP( ) procedure are MSISDN that represents call setup request and the day of request. If the condition in line (1) returns true then setup the call. Otherwise, fetch the record from HLR setup call and update $S_{i}$.

In line (1), $I$ is set to null. The statements in lines (2) and (3) compute the intersection of seven sets over the days from $\mathrm{fp}$ to $\mathrm{sp}$. Line (4) is for incrementing the day of seventh position by one. Line (5) is for copying intersection into seventh position. Line (6) is for incrementing the day of first position by one.

A simulation model is developed for evaluating the performance of SWSSD algorithm [4]. The definition of notation and the pseudocode for simulating over a given number of days is shown in Algorithm 3.

The metrics employed for evaluating the performance of the SWSSD algorithms are hit rate and throughput that shall be maximized.

The hit rate is the probability of the relevant record availability in VLR for processing a call setup request. The numbers of hits and call setup requests over a period of time are measured for computing hit rate. It is assumed that call setup requests are received randomly from a finite set of MSISDNs. The availability of relevant record of an MSISDN in VLR for processing its call setup request is referred to as a hit whereas nonavailability is referred to as a miss.

Throughput of a MSC is the number of call setups per unit time. The average call setup time for a call setup request is computed using

$$
\text { Average Call Setup Time }(\mathrm{ACST})=\frac{C_{1} H+C_{2} M}{H+M}
$$

where $C_{1}$ is call setup time in case of hit, $C_{2}$ is call setup time in case of miss, $H$ is number of hits over a period of time, and $M$ is number of misses over a period of time.

Obviously, $C_{2}$ is greater than $C_{1}$ as the relevant record is to be fetched from HLR in case of a miss. From the analysis of data pertaining to call setup times of Telephone Regulatory Authority of India (TRAI), $C_{1}$ and $C_{2}$ are assigned 3 and 7 seconds, respectively. 


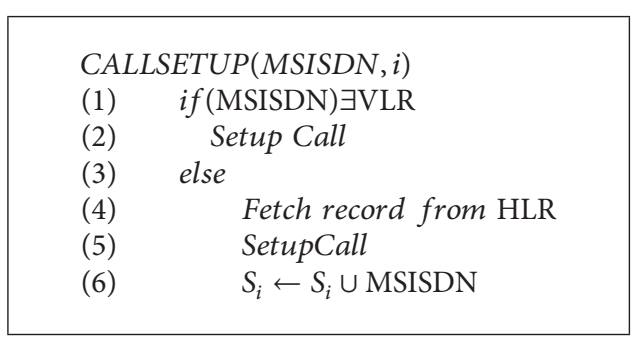

Algorithm 1: Call setup process.

\begin{tabular}{|c|c|}
\hline \multicolumn{2}{|c|}{$\operatorname{SWSSD}(f p, s p)$} \\
\hline & $I \leftarrow\{\emptyset\}$ \\
\hline & for $i=\mathrm{fp}$ to $\mathrm{sp} d o$ \\
\hline & $I \leftarrow I \cap S_{i}$ \\
\hline (4) & $\mathrm{sp} \leftarrow \mathrm{sp}+1$ \\
\hline (5) & $S_{\mathrm{sp}} \leftarrow I$ \\
\hline & $\mathrm{fp} \leftarrow \mathrm{fp}+1$ \\
\hline
\end{tabular}

Algorithm 2: Sliding window algorithm.

The reciprocal of average call setup time is throughput and the same is computed using

$$
\begin{aligned}
& \text { Throughput (TP) } \\
& =\frac{1}{\text { Average Call Setup Time (ACST) }} .
\end{aligned}
$$

A simulation model is developed for evaluating the performance of SWSSD algorithm. It is used to generate the performance measures of SWSSD algorithm over a period of 1001 days. It is evident from the results that there is a significant increase in performance metrics of the proposed SWSSD algorithm for one MSC service area comparing with Fixed Block of Seven Days (FBSD) algorithm [3]. Obviously, it is recommended to consider adopting the SWSSD algorithm for the entire GSM network for improving its throughput by $7.08 \%$.

\section{A Single Server Finite Queuing Model}

An entity that approaches a service facility for want of service is referred to as customer whereas the service facility is referred to as server. The service on arrival of a customer is commenced immediately provided the server is idle. Otherwise the customer has to wait in the queue until its turn. The six elements of a queuing model are interarrival, service times of customers, number of servers, queue discipline, maximum queue size, and calling population size.

The interarrival and service times of customers are probabilistic in nature following respective probabilistic distributions. The number of servers is either single or multiple. The identical multiple servers are arranged parallelly whereas the nonidentical multiple servers are arranged in tandem. The queue discipline specifies order in which the next customer is selected from the queue for providing service. The queue disciplines are First Come First Served (FCFS), Last Come First Served (LCFS), Selection in Random Order (SIRO), and order of priority. The maximum queue size is the maximum number of customers permitted to wait in queue. It is assumed as infinite whenever there is no restriction on queue size. The calling population size is assumed as infinite whenever it exceeds threshold.

A queuing model is descriptive in nature which provides the change of the state of a queuing system due to occurrence of customer arrival and departure events. The state of a queuing system is represented by the status of server (busy or idle), number of customers in system, and queue. As the arrival and departure events occur randomly over time, the state of the queuing system changes randomly.

The steady state performance measures of queuing system server utilization, mean number of customers in system/queue, and mean waiting time in system/queue can be computed. These measures are used in a cost model for deciding the number of servers and quality of service. All setup requests from mobile subscribers are customers whereas the MSC that can process one request at a time for providing call setup services is a server. Obviously, the number of mobile subscribers is finite. Hence, the queuing system under consideration is formulated as a single server finite queuing model.

\section{Integration of Sliding Window Algorithm with a Single Server Finite Queuing Model}

The sliding window algorithm ensures minimum average call setup time which is termed as average service time of queuing system. However, the sliding window algorithm does not consider the waiting of call setup request while a MSC is busy.

The average waiting time in queue shall be added to average call setup time to determine the average time a call setup request spends at a MSC. For this purpose, sliding window algorithm is integrated with a single sever finite queuing model. Henceforth, it is referred to as an integrated model depicted in Figure 2. Obviously, the integrated model is a single server finite queuing model. For processing a call setup request applies the SWSSD algorithm, which produces two deterministic call setup times $D_{h}$ and $D_{m}$ as a consequence of hit and miss, respectively. Therefore, the Kendall-Lee notation for representing the model is follows:

$$
M / D / 1:(\mathrm{FCFS} / \infty / \infty)
$$

It is assumed that the customer (call setup request) arrival rate follows Poisson distribution with parameter $\lambda$ denoted by $M$ whereas $D$ is a deterministic service time (call setup time) as defined below:

$$
D= \begin{cases}D_{h}, & \text { if record is available in VLR (hit) } \\ D_{m}, & \text { otherwise (miss). }\end{cases}
$$

Obviously, an analytical solution cannot be found for computing average waiting time of call setup requests in queue and it is to be determined through simulation. 


\section{$\operatorname{SWSSD}(\lambda, a, b, T)$}

$\lambda=$ Parameter of Poison Distribution

$a=1$ and $b=88243$ are parameters of discrete uniform distribution

$T$ = operating life of system in days

(1) $S_{i} \leftarrow\{\emptyset\}$ for $(1 \leq i \leq 7)$ : set of MSISDNs for $i$ th day, initially null set

(2) $\quad h \leftarrow 0$ : number of hits, initially zero

(3) $\quad i \leftarrow 1$

(4) $\quad j \leftarrow 1$

(5) for $i=1$ to 7 do

\{

(6) $\quad \mathrm{CR} \leftarrow$ Generate_Poison_Random_Variant $(\lambda)$

(7) for $k=1$ to $\mathrm{CR}$

\{

(8) $\quad s \leftarrow$ Generate_Discrete_Uniform_Random_variante $(a, b)$

(9) If profile of " $s$ " is found in VLR

\{

Setup call

\}

(10) Otherwise

\{

(a) Fetch the profile of " $s$ " to VLR from HLR

(b) Setup call

(c) $S_{i} \leftarrow S_{i} \cup s$

\}

\}

(11) $\quad$ Compute $I \leftarrow \bigcap_{i=1}^{7} S_{i}$ : Intersection of seven days

(12) Slide the window right by one day

(13) $\quad$ VLR $\leftarrow$ VLR $-S_{j}$ : VLR is updated

(14) $\quad j \leftarrow j+1$

(15) $\quad S_{i} \leftarrow I$

(16) While $(i \leq T)$ do

(17) $\quad \mathrm{CR} \leftarrow$ Generate_Poison_Random_Variant $(\lambda)$

(18) for $k=1$ to $\mathrm{CR}$

\{

(19) $\quad s \leftarrow$ Generate_Discrete_Uniform_Random_variante $(a, b)$

(20) If the profile of " $s$ " is found in VLR

\{

Setup call

$h \leftarrow h+1$

(21) Otherwise

\{

(a) Fetch the profile of "s" to VLR from HLR

(b) Setup call

(c) $S_{i} \leftarrow S_{i} \cup s$

\}

(22) Compute $I \leftarrow \bigcap_{k=j}^{i} S_{k}$ : Intersection of seven days from $j$ th day

(23) $\quad i \leftarrow i+1$ 


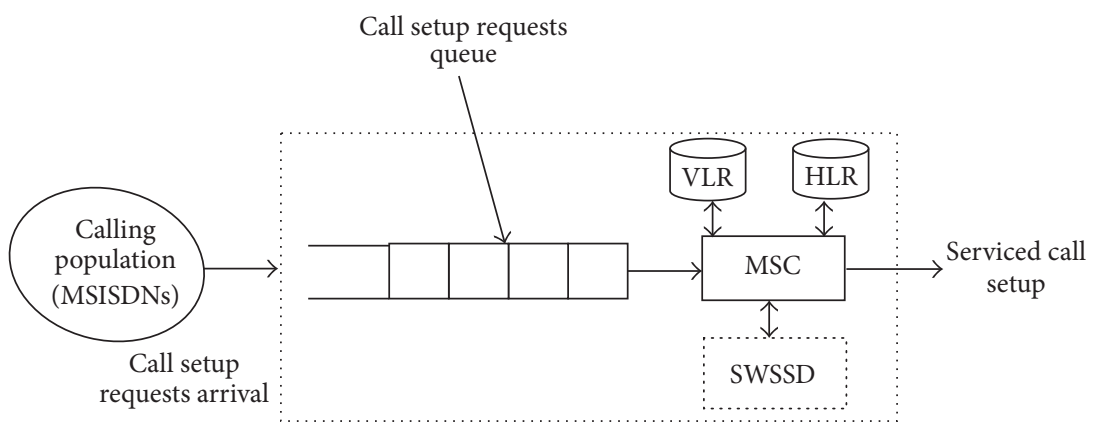

Figure 2: Integrated model.

\section{Simulation}

The simulation of the integrated model generates an artificial history of system facilitating the measurement of throughput of a MSC. The entities considered for developing simulation model are mobile stations and Mobile Switching Centre. The mobile stations are represented by their unique identities, MSISDNs. The call setup requests from MSISDNs are the customers. The Mobile Switching Centre (MSC) is a server. A set of mobile stations registered with a service provider constitute the calling population source from which call setup requests are received.

In GSM network, the profiles of MSISDNs comprising several attributes are stored in HLR at Gateway Mobile Switching Centre (GMSC). The profiles of MSISDNs in the service area of a MSC are stored in VLR. Whenever an MSISDN profile is not available in VLR for processing its call setup request, its profile is fetched from HLR. The deletion of the profile of an MSISDN from VLR depends on the sliding window algorithm.

The metric employed evaluating the performance of a MSC using integrated model is throughput considering waiting time in queue would be more realistic. Equations (6) and (7) define the average waiting time in the system and throughput, respectively;

$$
\text { AWTS }=\frac{W_{q}+D}{\text { No. of call setup requests }},
$$

where AWTS is average waiting time in system, $W_{q}$ is average waiting time in queue, $D$ is a deterministic service time, and $D=\left\{D_{h}\right.$, if record is available in VLR; $D_{m}$, otherwise $\}$.

Obviously, $D_{m}$ is greater than $D_{h}$ as the relevant record is to be fetched from HLR in case of its nonavailability. From the analysis of data pertaining to call setup times of Telephone Regulatory Authority of India (TRAI), $D_{h}$ and $D_{m}$ are assigned 3 and 7 seconds, respectively.

The reciprocal of average waiting time in system is throughput shown in

$$
\text { Throughput }(\mathrm{TP})=\frac{1}{\text { Average waiting time in system }} \text {. }
$$

The simulation is performed using TN3270 emulator for IBM (zSeries) mainframe on windows platform with z/OS
IBM mainframe operating system and DB2 database server. The notations and input parameters of simulation are shown in Table 1.

The output of simulation for 88243 call setup requests over 1001 days is presented in Table 2 . A call setup request has three attributes, namely, calls per day, MSISDN requesting call setup, and interarrival (time between two successive call setup requests) shown in columns (2), (3), and (4), respectively. The number of call setup requests per day is a random variate from Poisson distribution with parameter $\lambda$ $=87$.

A discrete uniform random variate represents the attribute MSISDN and its interarrival time is a random variate from an exponential distribution with parameter $1 / \mu=0.02$.

The SWSSD algorithm performs, whenever a subscriber arrives and makes a call setup request, the MSC checks its database to determine whether the record is available at its VLR. and If it is available, it returns "yes"; otherwise, it returns "no" shown in column (5). Accordingly, call setup service times are 3 and 7 for hit and miss, respectively, shown in column (6):

$$
\begin{aligned}
\mathrm{AT}_{i} & = \begin{cases}0 & \text { for } i=1 \\
\mathrm{AT}_{i-1}+\mathrm{IAT}_{i} & \text { for } i>1,\end{cases} \\
\mathrm{SB}_{i} & =\max \left(\mathrm{AT}_{i}, \mathrm{SB}_{i-1}\right), \\
\mathrm{SE}_{i} & =\mathrm{SB}_{i}+\mathrm{ST}_{i}, \\
\mathrm{WTQ}_{i} & =\mathrm{SB}_{i}-\mathrm{AT}_{i}, \\
\mathrm{WTS}_{i} & =\mathrm{SE}_{i}-\mathrm{AT}_{i} .
\end{aligned}
$$

The arrival time $\left(\mathrm{AT}_{i}\right)$ with respect to each call setup request is computed using (8) shown in column (7). Service Beginning Time $\left(\mathrm{SB}_{i}\right)$ and Service Ending Time $\left(\mathrm{SE}_{i}\right)$ with respect to each call setup request are computed using (9) and (10), respectively, shown in column (8) and column (9).

The waiting time in queue $\left(\mathrm{WTQ}_{i}\right)$ and waiting time in system $\left(\mathrm{WTS}_{i}\right)$ for each of the 88243 call setup requests 
TABLE 1: Simulation parameters.

\begin{tabular}{lcc}
\hline Symbol & Description & Value \\
\hline$N$ & Simulation run length in days & 1001 \\
$M$ & Calling population size & 88243 \\
$C$ & Ordered set of calling population & $\left\{\right.$ MSISDN $\left._{i} \in \mid 1 \leq i \leq 88243\right\}$ \\
$\lambda$ & Parameter of Poisson distribution, that is, mean number of call setup requests per day & 87 \\
$1 / \mu$ & Parameter of exponential distribution & 0.02 \\
$D_{h}$ & Call setup time in case of record available in VLR & 3 \\
$D_{m}$ & Call setup time in case of record unavailable in VLR & 7 \\
WS & Sliding window size in days & 7 \\
\hline
\end{tabular}

over 1001 days are computed and represented graphically in Figures 3, 4, and 5, respectively;

$$
\begin{aligned}
\mathrm{AWTQ} & =\frac{\sum \mathrm{WTQ}_{i}}{N}, \\
\mathrm{AWTS} & =\frac{\sum \mathrm{WTS}_{i}}{N} .
\end{aligned}
$$

Further, the numbers of hits are determined for each of the 143 blocks when the call setup requests are processed by employing sliding window of size seven days algorithm [4] for over a period of 1001 days. This model partitions the sliding window into seven blocks and maps onto seven days. The call setup request for each block of seven days is aggregated. The performance metrics such as hits, average call setup time, and throughput are computed for each block and the same is briefed in Table 3, when the call setup requests are processed by employing integrated model considering the waiting time in queue and service times are determined for each of the 143 blocks. Performance metrics such as average waiting time in queue, average waiting time in system, and realistic throughput are presented in Table 3 and the corresponding graphical representations are shown in Figures 6 and 7.

The performance metrics, average waiting time in queue (AWTQ) and average waiting time in system (AWTS) computed using (13) and (14), are 32.4552 and 32.5062, respectively. Accordingly, the throughput of a MSC is 0.03097 which is a reciprocal of AWTS, whereas the throughput of SWSSD algorithm without considering waiting in queue at a MSC is 0.18345 .

It is evident that the throughput of the proposed integrated model which considers waiting time in queue at a MSC is decreased by $83.12 \%$ for a single-channel MSC; it is a realistic measurement of throughput.

\section{Conclusions}

This study has proposed a realistic model for measuring throughput of a MSC integrating the sliding window algorithm with a single server finite queuing model. The sliding window algorithm minimizes the average call setup processing time and a single server finite queuing model determines the average call setup request waiting time in the queue. Hence, the reciprocal of the sum of average call setup processing time and average call setup request waiting time

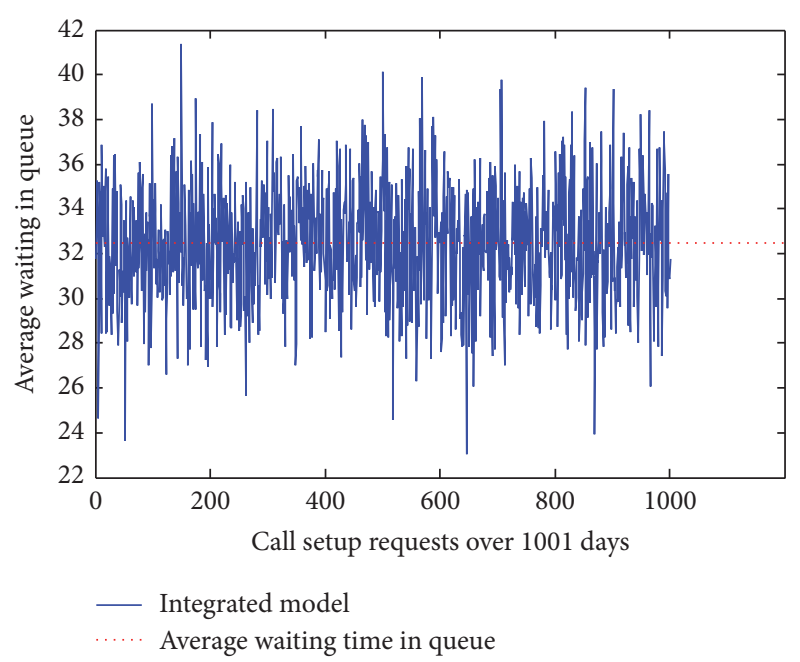

FIGURE 3: Waiting time in queue over 1001 days.

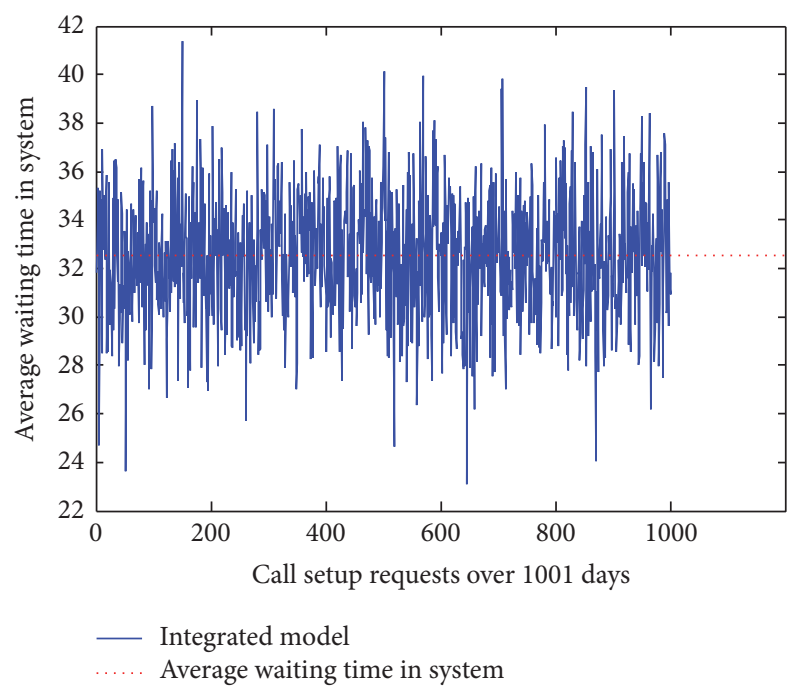

FIGURE 4: Waiting time in system over 1001 days.

in the queue is more realistic average throughput of a MSC under consideration. This model facilitates determining an optimal sliding window size.

However, the model assumes a MSC with a single channel for processing call setup requests. It is worth attempting to 


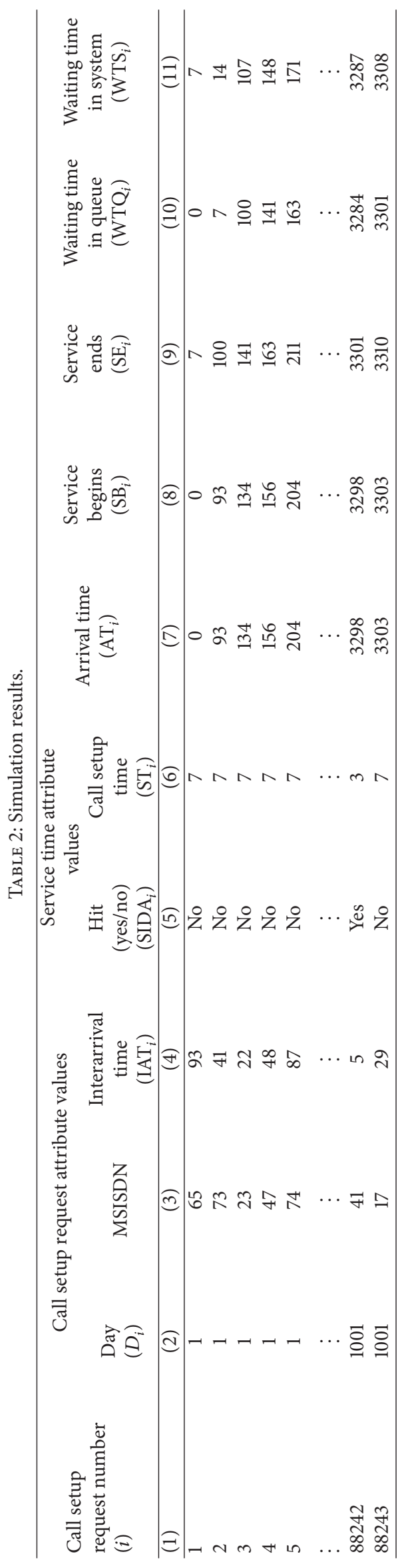


TABLE 3: Aggregated simulation results for each block.

\begin{tabular}{|c|c|c|c|c|c|c|c|}
\hline \multirow[b]{2}{*}{ Period/blocks } & \multirow[b]{2}{*}{$\begin{array}{l}\text { Call setup } \\
\text { requests }\end{array}$} & \multirow[b]{2}{*}{ Hits } & \multicolumn{2}{|c|}{ Sliding window algorithm } & \multicolumn{3}{|c|}{ Integrated model } \\
\hline & & & $\begin{array}{l}\text { Average call setup } \\
\text { time }\end{array}$ & Throughput & $\begin{array}{c}\text { Average } \\
\text { waiting time } \\
\text { in queue } \\
\text { (AWTQ) }\end{array}$ & $\begin{array}{l}\text { Average } \\
\text { waiting time } \\
\text { in system } \\
\text { (AWTS) }\end{array}$ & Throughput \\
\hline $1-7$ & 621 & 318 & 4.951691 & 0.2019 & 31.8776 & 31.9178 & 0.03133 \\
\hline $8-14$ & 549 & 262 & 5.091075 & 0.1964 & 39.1165 & 39.1621 & 0.02553 \\
\hline $15-21$ & 631 & 239 & 5.484945 & 0.1823 & 32.0570 & 32.1030 & 0.03115 \\
\hline $22-28$ & 616 & 244 & 5.415584 & 0.1846 & 31.5487 & 31.5957 & 0.03165 \\
\hline$\vdots$ & $\vdots$ & $\vdots$ & $\vdots$ & $\vdots$ & $\vdots$ & $\vdots$ & $\vdots$ \\
\hline $988-994$ & 638 & 241 & 5.489028 & 0.1821 & 33.5109 & 33.5501 & 0.02806 \\
\hline 995-1001 & 629 & 206 & 5.689984 & 0.1757 & 31.9793 & 32.0445 & 0.03120 \\
\hline
\end{tabular}

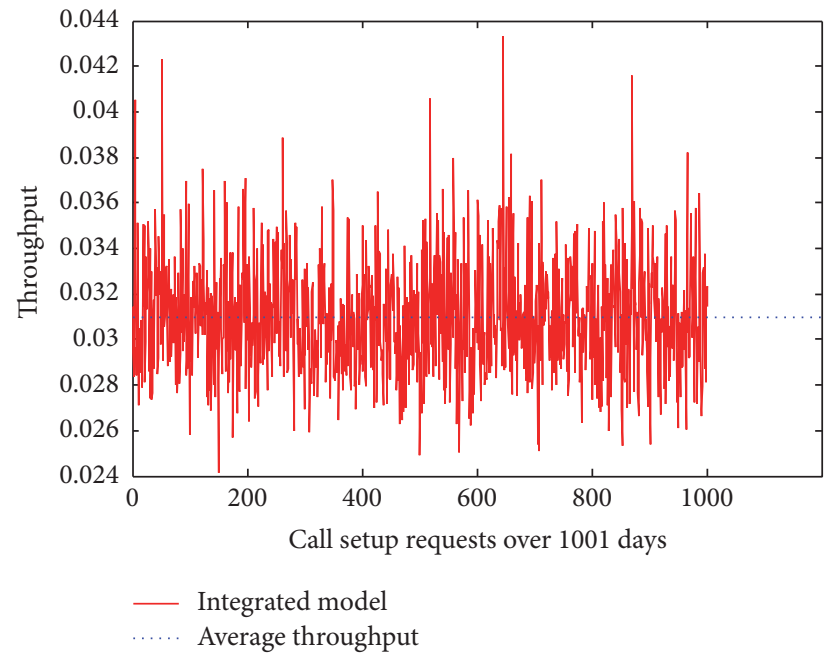

FIgURE 5: Throughput over 1001 days.

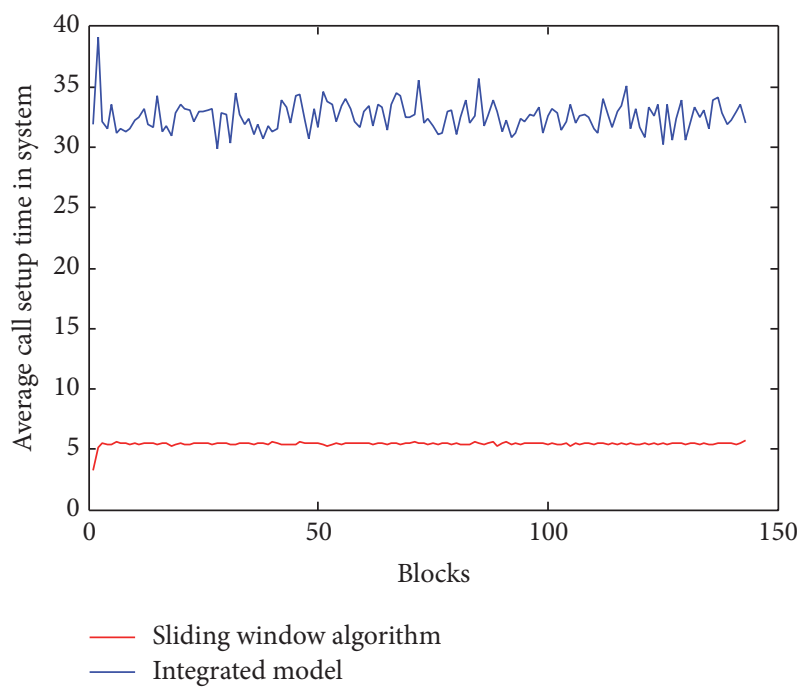

FIgURE 6: Call setup time in system versus blocks at a MSC.

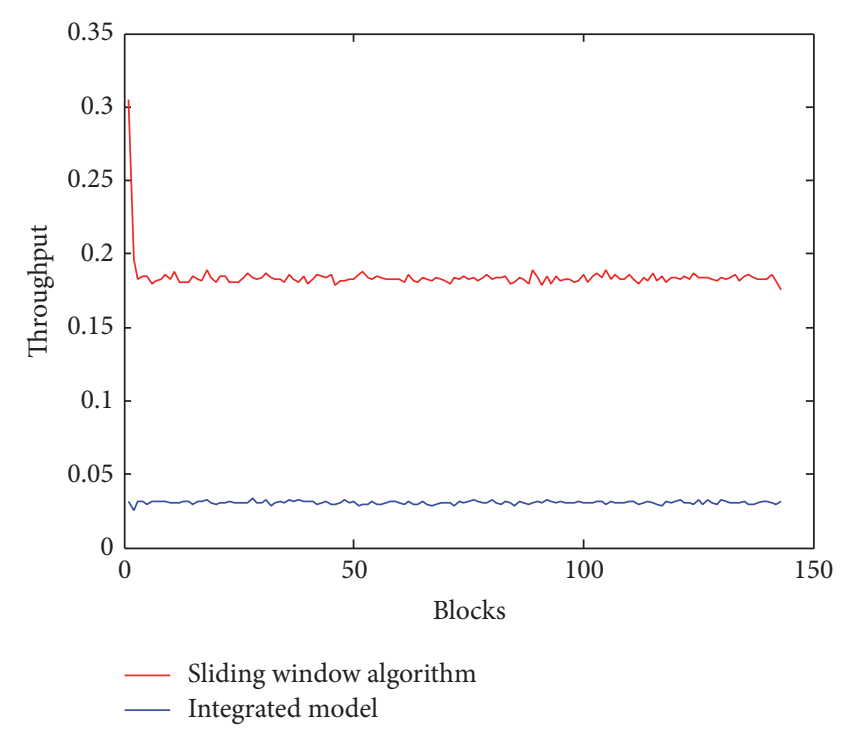

FIgURE 7: Throughput versus blocks at a MSC.

develop a model considering a MSC with multiple identical channels for processing concurrently multiple call setup requests for measuring still more realistic throughput of a MSC. Further, it facilitates formulating a cost model for determining an optimal number of channels with the criterion of maximizing throughput of a MSC. The study in this direction is under progress.

\section{Competing Interests}

The authors have no competing interests.

\section{Authors' Contributions}

The author of the article, Dinaker Babu Bollini, pursuing his doctoral programme from SV University, Tirupati, has conceptualized integrating queuing model with the Sliding 
Window of Seven Days Algorithm for investigating the effect of waiting times of call setup requests on throughput of a Mobile Switching Centre. The first co-author, Dr. Mannava Muniratnam Naidu, as supervisor of the doctoral work provided guidance in formulating and validating the conceptual simulation model, design of experiments, and analysis of simulation output. The second co-author, Dr. Mallikharjuna Rao Nuka, translated the conceptual simulation model into an operational simulation model and carried out the computer simulation.

\section{References}

[1] N. Mallikharjuna Rao and M. M. Naidu, "An intelligent location management approaches in GSM mobile network," International Journal of Advanced Research in Artificial Intelligence, vol. 1, no. 1, pp. 30-37, 2012.

[2] N. Mallikharjuna Rao and M. M. Naidu, "An Approach for Intelligent database maintenance of HLR and VLR databases," IACSIT International Journal of engineering and Technology, vol. 4, no. 5, pp. 532-536, 2012.

[3] S. H. Shah Newaz, A. Hossain, K. K. Al Zahid, and M. R. Amin, "Intelligence adaptation in visitor location register to enhance the performance of next generation cellular network," in Proceedings of the 2nd International Conference on Convergence Information Technology (ICCIT '07), pp. 1517-1522, IEEE, Gyeongju, South Korea, November 2007.

[4] M. R. Nuka and M. M. Naidu, "Sliding window method based on enhanced throughput prediction for improving GSM network efficiency by reducing HLR-VLR transitions," International Journal of Mobile Network Design and Innovation, vol. 5, no. 2, pp. 63-77, 2013.

[5] M. R. Nuka and M. M. Naidu, "An optimal sliding window size for maximising GSM network throughput," International Journal of Wireless and Mobile Computing, vol. 7, no. 5, pp. 509516, 2014.

[6] M. Sugano, D. S. Eom, M. Murata, and H. Miyahara, "Performance modeling for signaling traffic of wireless cellular networks," in Proceedings of 5th International workshop on Mobile Multimedia Communications (MoMuc '98), pp. 1-8, Berlin, Germany, 1998.

[7] K. Venkatachalam and P. Balasuramanie, "A hybrid resource allocation strategy with Queueing in Wireless Mobile Communication Networks," Journal of Computer and Information Science, vol. 2, no. 3, pp. 3-14, 2009.

[8] D. Tipper, T. Dahlberg, H. Shin, and C. Charnsripinyo, "Providing fault tolerance in wireless access networks," IEEE Communications Magazine, vol. 40, no. 1, pp. 58-64, 2002.

[9] S. S. Moghaddam and A. H. Khodadadi, "A novel measurement based channel assignment method," in Proceedings of the International Symposium on Telecommunications (IST'05), pp. 10231028, Shiraz, Iran, 2005.

[10] A. Z. Melikov and A. T. Babayev, "Refined approximations for performance analysis and optimization of queueing model with guard channels for handovers in cellular networks," Journal of Computer Communications, vol. 29, no. 9, pp. 1386-1392, 2006.

[11] T. Salih and K. Fidanboylu, "Modeling and analysis of queuing handoff calls in single and two-tier cellular networks," Computer Communications, vol. 29, no. 17, pp. 3580-3590, 2006.
[12] V. Jindal and S. Dharmaraja, "Call processing delay analysis in cellular networks: a queuing model approach," OPSEARCH, vol. 46, no. 3, pp. 289-302, 2009.

[13] J. khan, "Handover management in GSM cellular system," International Journal of Computer Applications, vol. 8, no. 12, pp. 14-24, 2010.

[14] T. Bonald, "Insensitive queuing models for communication networks," in Proceedings of the 1st International Conference on Performance Evaluation Methodologies and Tools (VALUETOOLS '06), pp. 458-467, Pisa, Italy, October 2006.

[15] E. Caushaj, I. Ivanov, H. Fu, I. Sethi, and Y. Zhu, "Evaluating throughput and delay in $3 \mathrm{G}$ and $4 \mathrm{G}$ mobile architectures," Journal of Computer and Communications, vol. 2, no. 10, pp. 1-8, 2014.

[16] E. Sayawu and Y. Diaba, "Evaluating the effect of FIFO queuing scheme on originating and handoff calls in cellular networks," International Journal of Advanced Research in Computer and Communication Engineering, vol. 4, no. 7, pp. 581-585, 2015. 


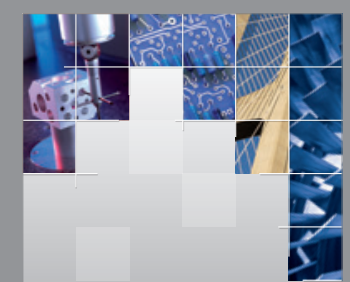

\section{Enfincering}
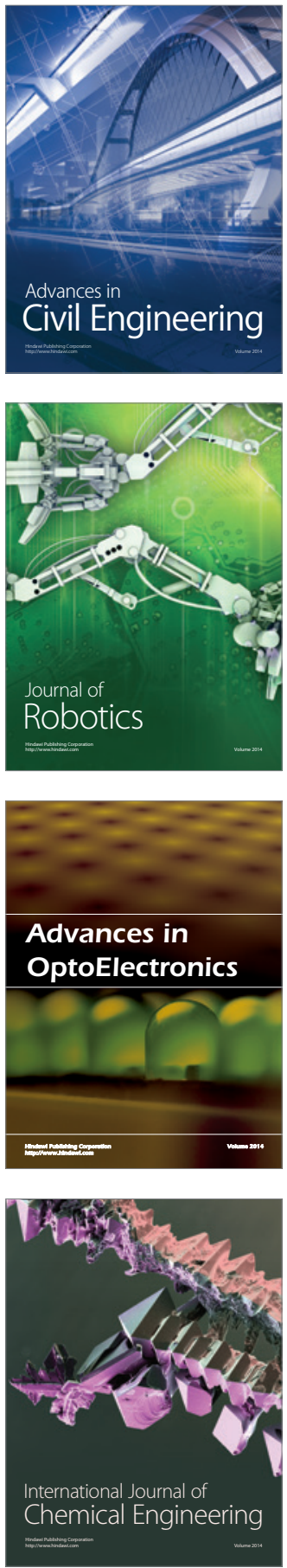

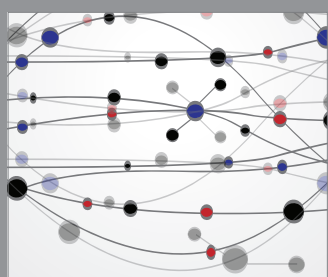

The Scientific World Journal

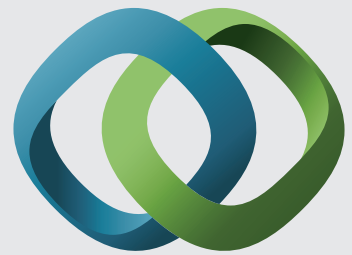

\section{Hindawi}

Submit your manuscripts at

http://www.hindawi.com
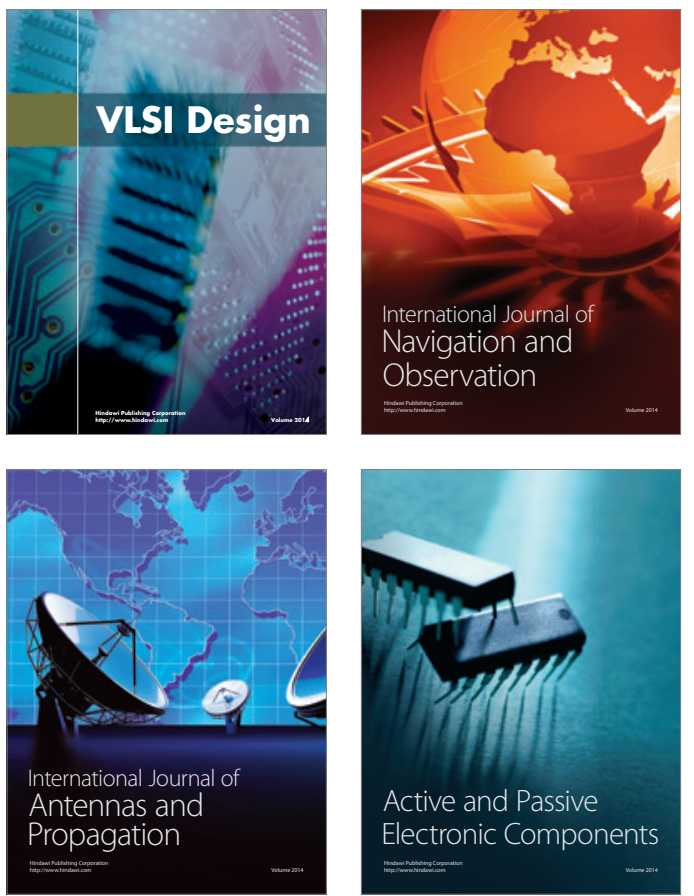
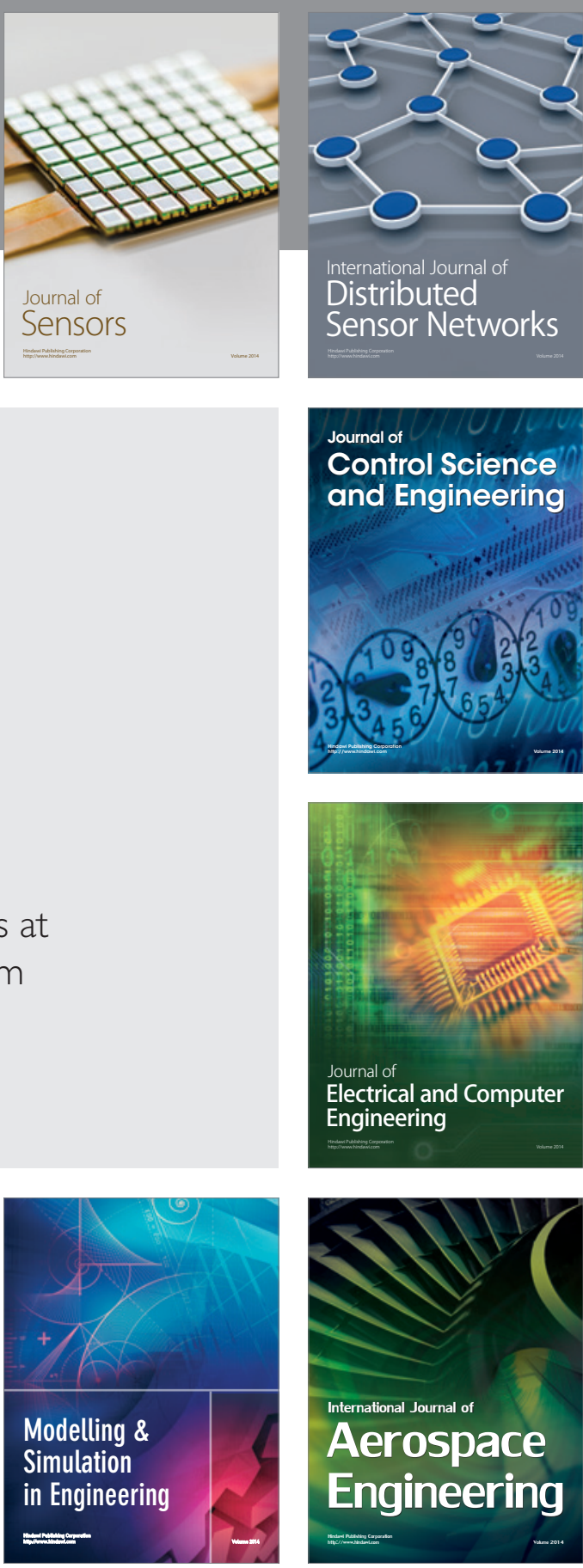

International Journal of

Distributed

Sensor Networks

Journal of

Control Science

and Engineering
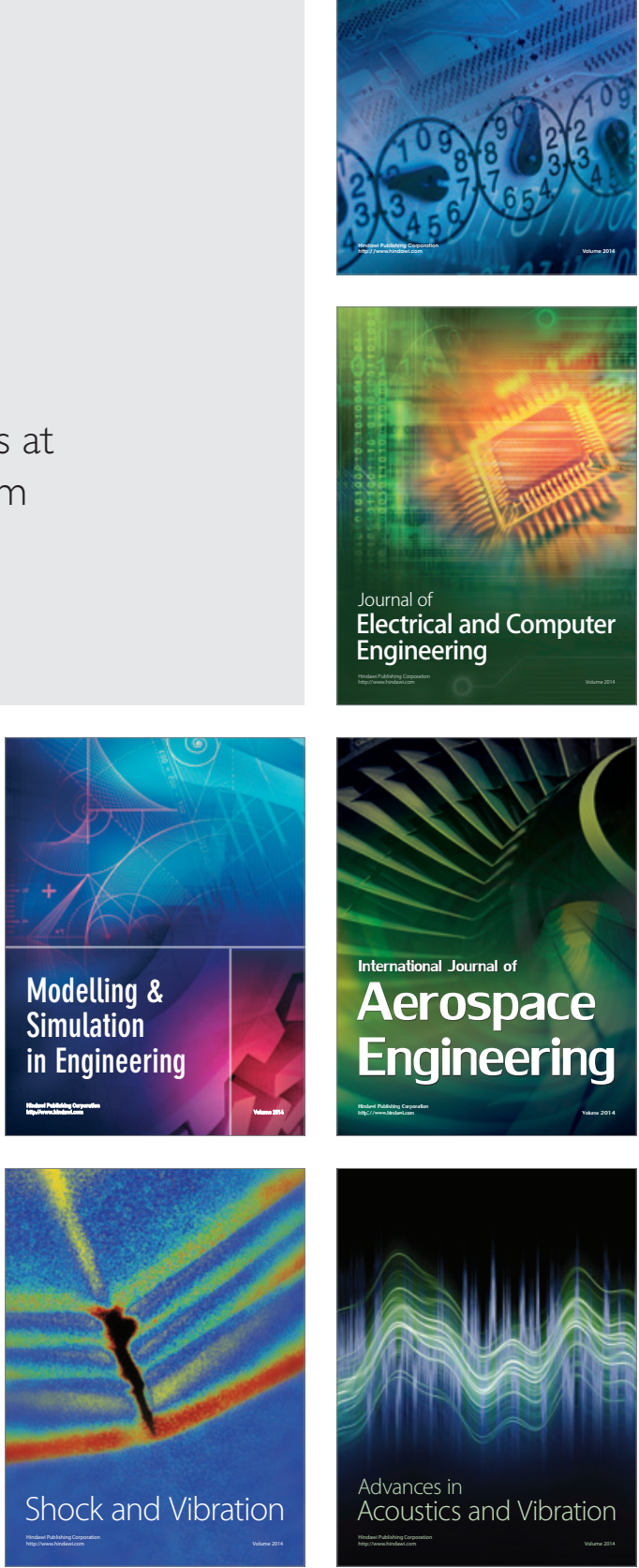